\title{
Percutaneous Treatment of Renal Cystic Nephroma
}

\author{
ATREYA DASH, M.D., and J. STUART WOLF, JR., M.D.
}

\begin{abstract}
Cystic nephroma is a rare, presumed benign, renal tumor that occurs in both children and adults. When its presence is suspected preoperatively, nephron-sparing surgery can be applied. We describe treatment of a cystic nephroma that is the first reported, to our knowledge, using endoscopic techniques.
\end{abstract}

\section{INTRODUCTION}

$\mathbf{C}^{2}$ YSTIC NEPHROMA is an unusual, presumed benign, renal tumor that occurs in both children and adults. The usual treatment until recently has been radical nephrectomy. We report a case that was treated using endoscopic techniques to preserve renal function.

\section{CASE REPORT}

A 53-year-old woman with no significant medical history and normal renal function was found to have an incidental right renal mass extending into the collecting system on a CT scan obtained to evaluate right upper-quadrant discomfort. Radical nephrectomy was recommended, but she presented to our clinic for a second opinion regarding less-invasive options, by which time, the presenting discomfort had resolved. The CT scan revealed an unusual mass with cystic components and extension into the renal pelvis (Fig. 1). Voided-urine cytology study was negative. With diagnostic flexible ureteroscopy, we noted a large, smooth, multiloculated mass that appeared to grow out from a middle calix. Biopsies were taken using a $3 \mathrm{~F}$ cup biopsy device passed through the flexible ureteroscope, and a 3F flexible biopsy needle was used to puncture the wall of the mass. An aspirate of fluid from inside the mass and renal pelvic barbottage were submitted for cytologic analysis. Examination of the other calices demonstrated no abnormalities. The biopsy was interpreted as urothelium with granulomatous inflammation. The aspirate and pelvic wash contained atypical cells that were not diagnostic. Because laboratory analyses did not diagnose obvious malignancy, and on the basis of the ureteroscopic and radiologic appearance, we suspected an unusual intrapelvic extension of a benign cystic lesion. The patient requested the least-invasive approach possible to firmly establish the diagnosis.

We therefore performed percutaneous endoscopic resection of the portion of the mass within the collecting system using a $24 \mathrm{~F}$ resectoscope through a single upper-pole access tract. The endoscopic appearance was of a smooth-surfaced multilocular mass that had a spongy cut edge on resection, with unremarkable vascularity. The hematocrit on postoperative day 1 was $38.4 \%$ compared with $42.7 \%$ preoperatively. The resection and recovery were uncomplicated. Pathologic interpretation was cystic nephroma. A follow-up CT urogram 4 weeks later confirmed excision of the intrapelvic component of the mass (Fig. 2).

\section{DISCUSSION}

Cystic nephroma is a rare renal neoplasm characterized by multilocular cysts lined with epithelium but separated by fibrous septa. The cysts do not communicate with the collecting system. The lesion usually arises from the renal parenchyma, but cases with a predominant component within the collecting system have been reported. ${ }^{1,2}$ Two recent case reports documented curative excision of cystic nephroma involving the collecting system using open nephron-sparing surgical techniques, ${ }^{1,2}$ although partial nephrectomy had previously been performed for a parenchymal mass in a solitary kidney. ${ }^{3}$ To our knowledge, our case represents the first successful treatment of cystic nephroma using a percutaneous endoscopic approach. We have gained expertise in performing percutaneous resection of urothelial tumors within the collecting system $^{4}$ and applied our experience in this case. We believe that the percutaneous approach is also appropriate in treating cystic nephroma when the mass predominantly involves the collecting system and there is a strong suspicion that the lesion will be benign. To our knowledge, our case represents the first treatment of cystic nephroma using a percutaneous endoscopic approach. Long-term follow-up imaging will be obtained because the natural history of cystic nephroma has not been well defined. Even with this apparently benign lesion, growth might well occur. If there is radiologic evidence of growth, we will recommend par-

\footnotetext{
Department of Urology, University of Michigan, Ann Arbor, Michigan.
} 


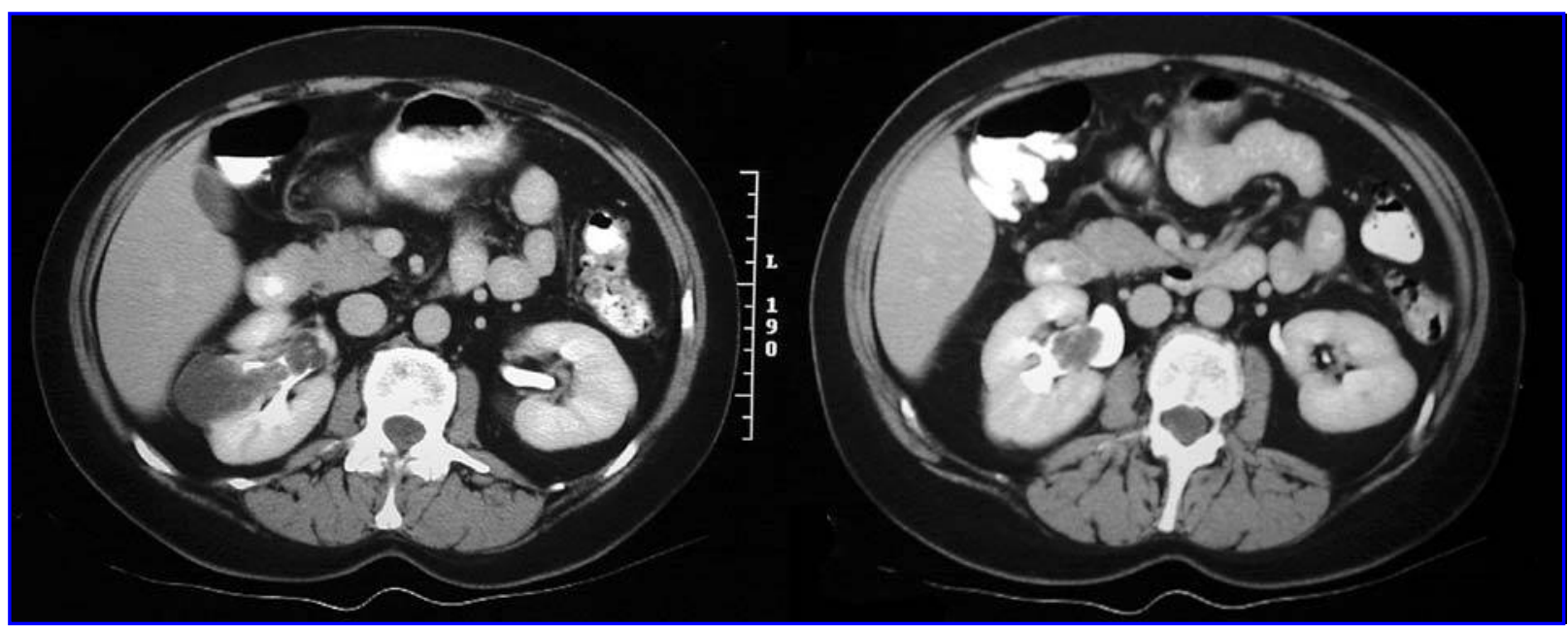

FIG. 1. Two CT images reveal unusual mass with both extension into renal pelvis and cystic components.

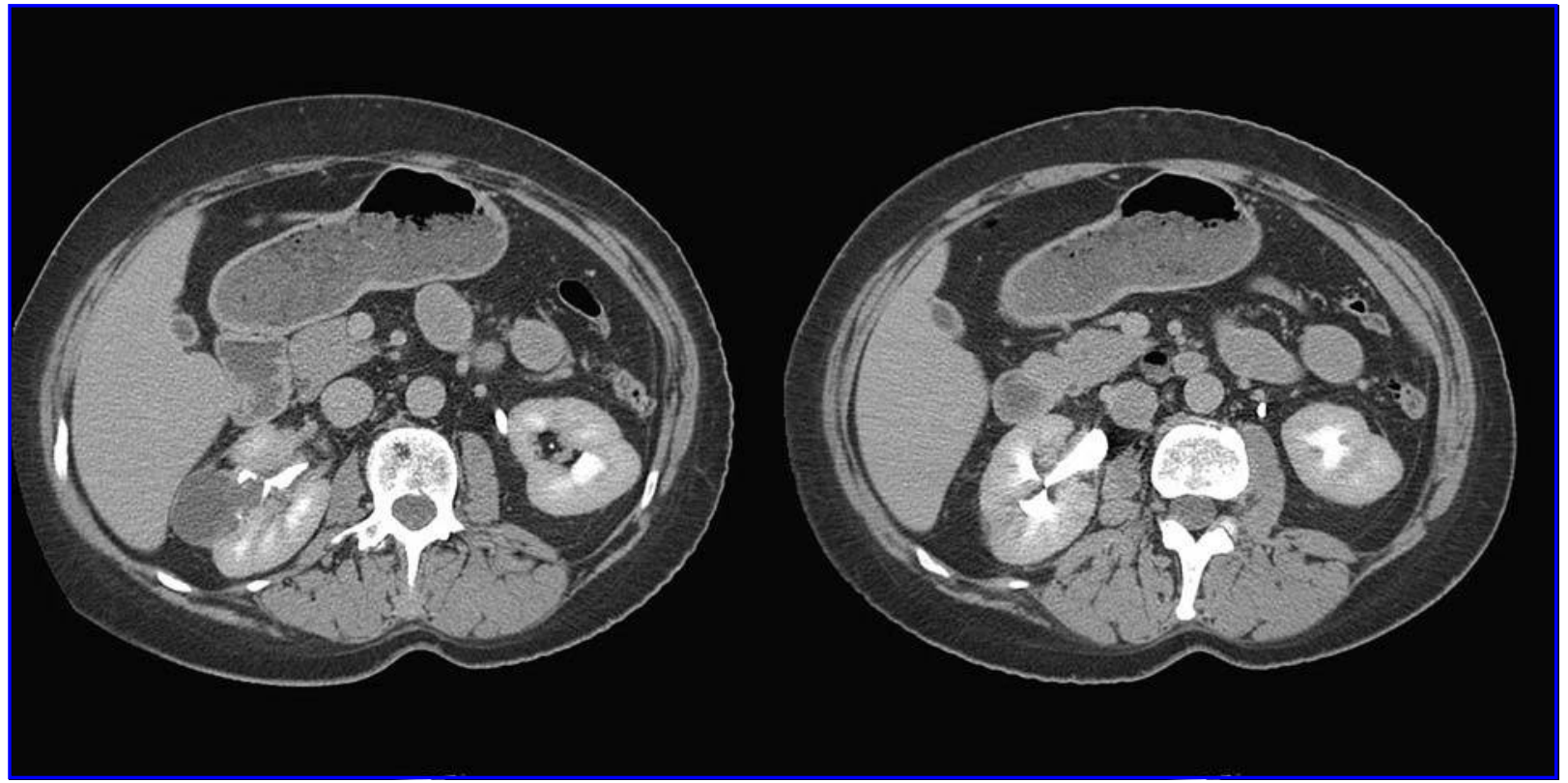

FIG. 2. Postprocedure CT images confirm resection of portions of mass that had extended into collecting system.

tial nephrectomy, given the unknown outcome of persistent cystic nephroma tissue.

\section{REFERENCES}

1. Kural AR, Obek C, Ozbay G, Onder AU. Multilocular cystic nephroma: An unusual localization. Urology 1998;52:897-899.

2. Gettman MT, Segura JW. An unusual case of multilocular cystic nephroma with prominent renal pelvis involvement treated with nephron sparing techniques. J Urol 1999;162:482.

3. Ferrer FA, McKenna PH. Partial nephrectomy in a metachronous multilocular cyst of the kidney (cystic nephroma). J Urol 1994;151: $1358-1360$
4. Suh RS, Faerber GJ, Wolf JS Jr. Predictive factors for applicability and success with endoscopic treatment of upper tract urothelial carcinoma. J Urol 2003;170:2209-2216.

Address reprint requests to: J. Stuart Wolf, Jr., M.D. Dept. of Urology University of Michigan TC 2916, Box 0330 1500 East Medical Center Drive Ann Arbor, MI 48109

E-mail:wolfs@umich.edu 
This article has been cited by:

1. J. Stuart Wolf, Jr. , Atreya Dash . 2008. Failure of Percutaneous Endoscopic Resection of a Renal Cystic Nephroma on Longer-Term Follow-UpFailure of Percutaneous Endoscopic Resection of a Renal Cystic Nephroma on Longer-Term Follow-Up. Journal of Endourology 22:7, 1505-1508. [Abstract] [PDF] [PDF Plus] 